\title{
Diseño conceptual de audífonos de conducción ósea y accionamiento cutáneo
}

\author{
Fuentes Jorge Mauricio ${ }^{1}$ \\ ORCID: https://orcid.org/0000-0003-0342-643X \\ jmfuentes@uce.edu.ec \\ Universidad Central del Ecuador \\ Quito Ecuador
}

Villalobos Andrea ${ }^{3}$

ORCID: https://orcid.org/0000-0002-3552-9030

avillalobos@uce.edu.ec

Universidad Central del Ecuador

Quito Ecuador

\author{
Yánez Henry ${ }^{2}$ \\ ORCID: https://orcid.org/0000-0002-1611-3697 \\ hayanez@uce.edu.ec \\ Universidad Central del Ecuador \\ Quito Ecuador \\ Veintimilla Diego \\ ORCID: https://orcid.org/0000-0003-4331-8388 \\ Diego Veintimilla \\ dveintimilla@outlook.com \\ Solidarity Tech \\ Quito Ecuador
}

Recibido (08/02/21), Aceptado (18/02/21)

\begin{abstract}
Resumen: En el Ecuador los problemas de hipoacusia generan en los niños que la padecen graves problemas de comunicación, al ser este un problema de más de 1000 casos al año, se requiere una solución de bajo costo. Actualmente para solucionar problemas de hipoacusia causados por daños en el oído externo, se usan en casos audífonos de conducción ósea, que son dispositivos que se colocan mediante implante o mediante cintillos unidos a la cabeza, junto al hueso mastoides, este tipo de dispositivos generan rechazo en niños, debido a su incomodidad y evidente notoriedad. En este proyecto se hace uso de la metodología de la Ingeniería Kansei (IK), enfatizando en las emociones para el desarrollo de un dispositivo que cause respuestas positivas en los niños que lo usan y también en sus padres. Para aplicar el método de IK se plantearon 16 diseños de dispositivos de conducción ósea y mediante un análisis estadístico de la teoría de cuantificación se seleccionó un diseño que genere las emociones definidas en los usuarios. El dispositivo diseñado, luego de la evaluación realizada cubre las expectativas de los niños y genera una aceptación por parte del usuario.
\end{abstract}

Palabra Claves: Kansei, audífono, conducción ósea, diseño de producto.

\section{Conceptual design of bone conduction and skin drive hearing aids}

\begin{abstract}
In Ecuador, hearing loss problems cause serious communication problems in children with severe communication problems, being a problem of more than 1000 cases per year, a low-cost solution is required. Currently to solve problems of hearing loss caused by damage to the external ear, they are used in cases bone conduction hearing aids, which are devices that are placed by implant or by belts attached to the head, next to the mastoid bone, this type of devices generate rejection in children, due to their discomfort and visible notoriety. This project makes use of the Kansei Engineering (IK) methodology and emotional design for the development of a device that causes positive emotions in children who use it and in their parents. To apply the IK method, 16 designs of bone conduction devices were developed, and a statistical analysis of quantification theory selected a design that generates the emotions defined in the users. The designed device, after the evaluation carried out covers the expectations of the children and does not cause the rejection.
\end{abstract}

Keywords: Kansei, earphone, bone conduction, product design.

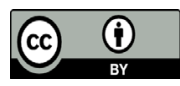




\section{I.INTRODUCCIÓN}

La Organización Mundial de la Salud (OMS) especificó que más del 5\% de la población mundial, es decir 360 millones de personas, padece pérdida de audición discapacitante. Entre ellos, 328 millones son adultos y 32 millones niños [1]. El Estudio Colaborativo Latino Americano de Malformaciones Congénitas (ECLAMC) recopila información de los recién nacidos que padecen malformaciones como es el caso de la microtia (malformación auditiva congénita que da lugar a una oreja pequeña) la cual provoca la pérdida de la capacidad auditiva produciéndose una dificultad o imposibilidad para oír normalmente (hipoacusia), este estudio y recopilación de información lleva establecido ya 23 años [2], ha encontrado que la tasa de niños que tienen malformaciones como la microtia va aumentado constantemente y uno de los factores es la altura, ya que se encuentra una aparición de la de la enfermedad en el $86 \%$ de niños con microtia que reside sobre los 2500 metros sobre el nivel del mar, siendo la ciudad de Quito quien abarca el 75\% de la población [3].

Se dice que alguien sufre pérdida de audición cuando no es capaz de oír tan bien como una persona cuyo sentido del oído es normal, es decir, cuyo umbral de audición en ambos oídos es igual o superior a 25 dB. La pérdida de audición puede ser leve, moderada, grave o profunda. Afecta a uno o ambos oídos y entraña dificultades para oír una conversación o sonidos fuertes [3]. La prevalencia mundial de la hipoacusia se sitúa en 5/1000 recién nacidos vivos, lo que supone que alrededor de 1500 niños nacen en el Ecuador con algún grado de hipoacusia. Cabe destacar que sólo una quinta parte de ellos, es decir 1/1000, presentan hipoacusia severa profunda y lo que representa en el Ecuador unos 300 casos nuevos cada año [5].

Viendo esta problemática, se desarrolló un dispositivo de audio por conducción ósea que beneficiará a niños y niñas de 5 a 13 años de edad, luego de un análisis estadísticos de clúster, con hipoacusia debido a factores como malformaciones congénitas de oído externo y medio, otitis crónica y así mismo un sin número de enfermedades que provoca esta discapacidad. Se desarrollo un producto que sin duda mejore las condiciones de vida de este grupo de niños. Comprende la interacción de diferentes campos del conocimiento como el diseño industrial, la electrónica, la medicina y la acústica para integrarlo de manera adecuada.

En un primer momento de la investigación, se recopilaron para el diseño: datos médicos, archivos de tomografías médicas, libros de texto, artículos científicos, tesis, documentos web que puedan sustentar el proyecto. Posteriormente se realizaron entrevistas con médicos especialistas relacionados al tema, profesores, padres de familia y usuarios finales con el fin de obtener los requisitos y objetivos de diseño.

\section{A.Estado del Arte}

El área de la salud ha evolucionado de manera significante pues día a día se va creando y descubriendo soluciones ante los distintos padecimientos de las personas provocados por enfermedades, esto se ha dado por la interacción de distintos campos con es la tecnología y sin duda el diseño industrial que brindan grandes aportes para la invención e innovación de nuevos dispositivos que mejoren la calidad de vida las personas. Muchos avances técnicos y tecnológicos que ayudan a niños y niñas con deficiencia auditiva a integrarse a la sociedad y a eliminar las barreras existentes en un mundo hecho para normo-oyentes, optimizando su calidad de vida. Dentro de un sin número de alternativas de dispositivos que se generan a diario en el mundo se puede mencionar a los audífonos que, aunque no es una solución definitiva para eliminar la deficiencia auditiva puede sin duda mejorar o corregir la audición de los deficientes auditivos [5].

Un auxiliar auditivo consta de uno o varios micrófonos que captan la señal sonora, esta información es transmitida al amplificador ubicado en la parte interna de la prótesis auditiva y ésta señal amplificada es llevada al oído externo muy cerca de la membrana timpánica a través del molde auditivo y desde ahí la fuente sonora continúa el mismo trayecto que se realizaría en una persona con 12 audición normal, a diferencia que la señal tiene un mayor volumen para que pueda ser percibida por el niño con deficiencia auditiva [5].

Los implantes cocleares son otra opción para intentar solucionar la hipoacusia, consiste en una prótesis eléctrica que pretende paliar una deficiencia bilateral del oído interno, sea profunda o severa, adquirida o congénita. Contrariamente a las prótesis auditivas acústicas, que actúan por medio del órgano de Corti, los implantes cocleares estimulan directamente las neuronas auditivas [6]. De igual forma otra alternativa es sin duda son los audífonos por conducción ósea el cual es un implante de conducción ósea es un dispositivo médico semi implantable que transmite sonido por conducción ósea directa al oído interno, evitando el oído externo y el oído medio. Consta de un procesador externo con un micrófono que capta el sonido y lo transmite al implante, que hará vibrar el hueso del cráneo y los líquidos del oído interno, empleando la capacidad natural del cuerpo para transmitir el 
sonido a través del tejido óseo [7].

En este trabajo se estudió la influencia del diseño de un dispositivo de conducción ósea en la percepción de las emociones de niños con hipoacusia leve y moderada, con el objeto de diseñar un audífono que no cause rechazo en niños y padres. Para realizar esto se empleó la ingeniería Kansei, técnica de desarrollada por [8] y que se ha usado para el desarrollo de diferentes tipos de productos como interfases de usuario [9], productos seriados [10], [11]y hasta en el área arquitectónica [12], [13]. En la primera parte se presentan los fundamentos teóricos y matemáticos de la ingeniería Kansei, luego en la metodología se explica cómo se aplicó la ingeniería Kansei para el desarrollo de unos audífonos de conducción ósea. En la sección de resultados se presenta la evaluación realizada con el método de cuantificación QT1 [14] y regresión lineal con variables ficticias (dummy) de cada una de las palabras Kansei definidas. También se presenta una evaluación final del diseño seleccionado, realizando una evaluación de la aceptación estética a niños con hipoacusia. Cabe indicar que el artículo se centra en la parte estética del producto, los estudios de mejora auditiva se encuentran en otro artículo.

\section{II.DESARROLLO}

\section{A.Ingeniería Kansei y emociones}

La Ingeniería Kansei nace en Japón en los años 70 a través del trabajo de Mitsuo Nagamachi. El propio autor define la IK como "una tecnología ergonómica para el desarrollo de productos orientados a las necesidades y sentimientos del consumidor/usuario". Este método se centra en los deseos y necesidades emocionales de los consumidores [15], [16]. A grandes rasgos, aplicando la Ingeniería Kansei, se consigue obtener información acerca de las emociones que produce un producto al usuario y traducir dichas emociones o sentimientos en propiedades estéticas del producto [4]. Las emociones que causa el producto en los usuarios se pueden medir usando técnicas biométricas [17], [18] y técnicas de procesamiento de imagen [19] así como también métodos más sencillos como encuestas. Esto se consigue mediante el sistema de IK, el cual utiliza métodos estadísticos que aseguran la validez de los resultados, en la Fig. 4 se muestra un esquema del proceso de la IK.

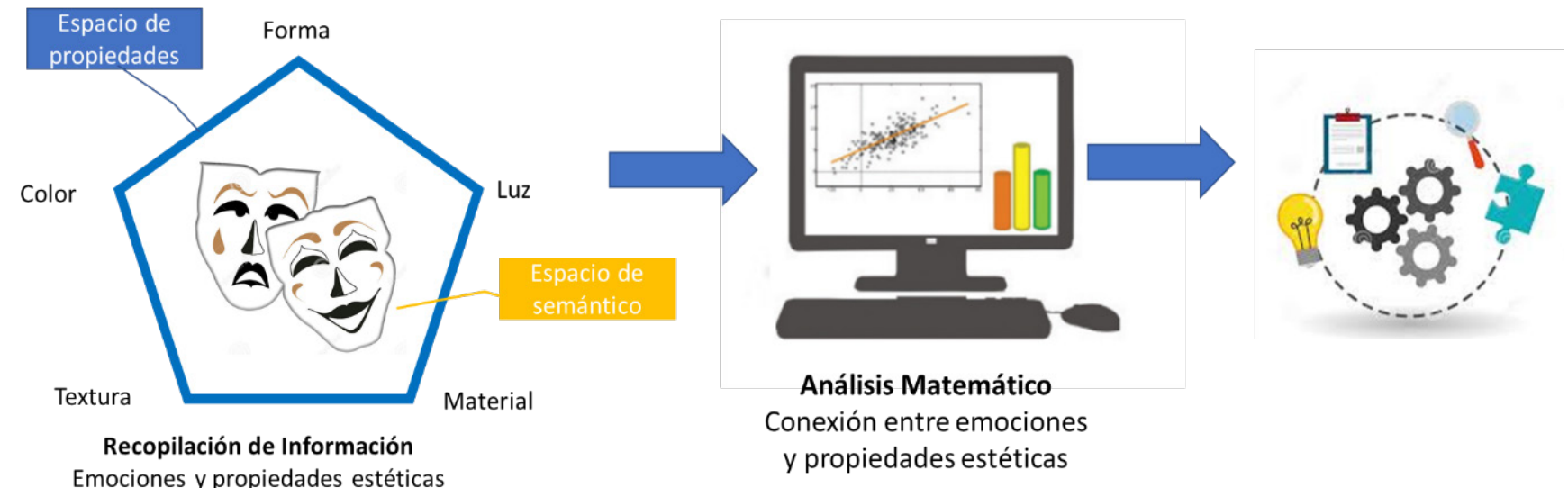

Fig.1 Esquema de la metodología de la Ingeniería Kansei.

Los pasos detallados para aplicar el método de la IK se muestran en la Fig.2, los cuales se describen en los siguientes párrafos. 


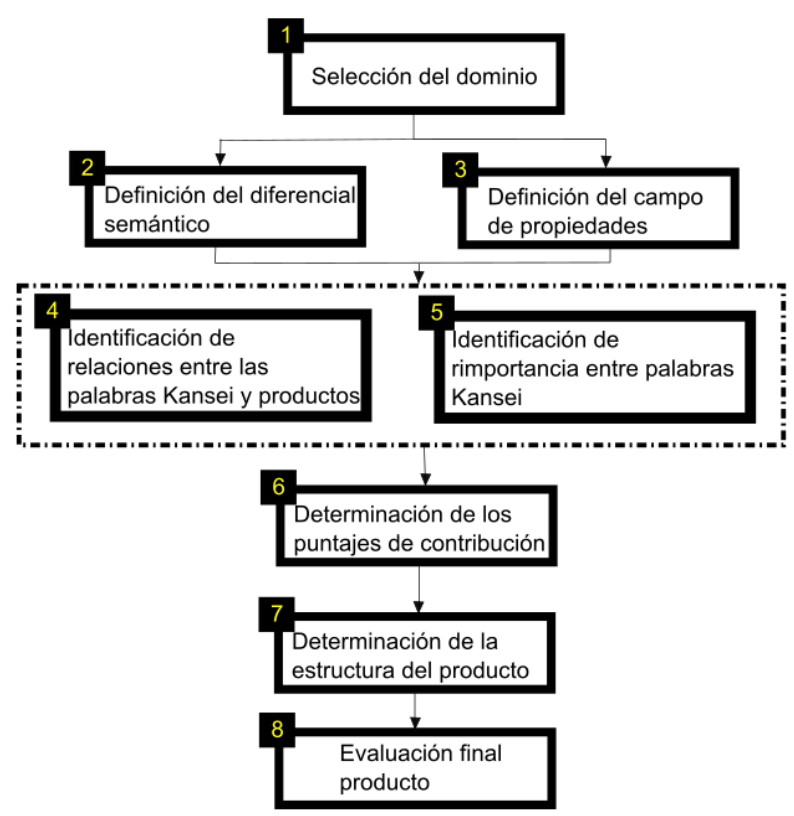

Fig.2 Esquema detallada de la implementación de la metodología de la Ingeniería Kansei (IK)

\section{B.Selección del dominio}

En este paso se decide sobre qué área de estudio se investigará, lo cual debe estar acorde con las áreas de mercado de la empresa o institución y sus productos actuales y su estrategia de negocio, es decir se debe definir en qué mercado se estudiará la solución de diseño.

\section{Análisis Clúster}

El objetivo del análisis clúster es para separar al conjunto de encuestados en grupos homogéneos, para esto en la encuesta se solicitan datos como la edad, preferencias de usos del producto, localización geográfica, conocimiento del producto o cualquier otra variable que podría diferenciar el rango de respuestas obtenidas al evaluar las palabras Kansei. Con estas encuestas se podrá establecer perfiles de usuarios con relación a las preguntas realizadas al evaluar el diferencial semántico.

\section{C.Definición del campo semántico}

El campo semántico define el conjunto de emociones o "Kanseis" que genera el producto en los usuarios. Para esto se requiere recopilar las sensaciones que generarán los productos en los usuarios. Inicialmente se recopilan información de las sensaciones y emociones y propiedades estéticas que causa un producto en el usuario o cliente, a través de la revisión de revistas especializadas, catálogos de productos, publicidad, entrevistas con expertos y usuarios, con lo cual se define un espació semántico, inicialmente se pueden tener cientos de sensaciones y emociones que puede causar un producto, las cuales son reducidas, aplicando métodos con el diagrama de afinidad o método KJ [20].

En este estudio se usará la IK, tipo I, usando el método la escala de diferencial semántico de Osgood. Este método se utiliza para aclarar la estructura del lenguaje psicológico. Osgood organizó palabras positivas y negativas a ambos lados de una línea horizontal. Por ejemplo, hermoso-feo se establecen en ambos lados de un continuo. Pero la IK está destinada a lograr un buen diseño, no un diseño feo. Por lo tanto, organizamos palabras Kansei positivas y negativas en ambos lados de la escala, como hermosas, no hermosas. Hay varias escalas, 5 escalas, 7 escalas, 9 escalas y 11 escalas [21], ver en la Fig. 3, un ejemplo de la escala de diferencial semántico (DS). 


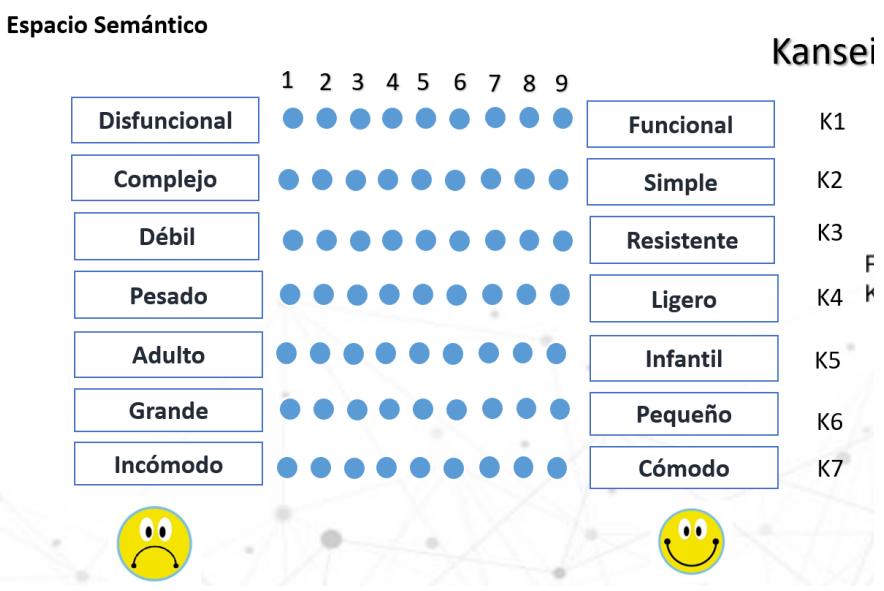

Fig. 3. Ejemplo de escala de diferencial de Osgood para las palabras Kansei definidas para un audífono.

\section{D.Definición del campo de propiedades}

El campo de propiedades incluye la identificación de todas las propiedades del producto con las que se quieren producir determinadas emociones en el usuario, para esto se divide al producto en sus propiedades físicas características, esto se lo puede realizar con la participación de los expertos en el producto y diseñadores y también revisando información de la cartera de productos de la empresa. Cada una de las propiedades poseerá una serie de subniveles, por ejemplo, la propiedad cuerpo poseerá los subniveles rectangular, redondo, paralelogramo y ovalado. Aquí generalmente se procede en base a la experiencia del equipo de diseño o usando ingeniería inversa analizando productos similares en el mercado.

En esta parte del proceso también se realizan diferentes combinaciones sub-propiedades que reflejen todas o la mayoría de las combinaciones de las propiedades estéticos definidas en el campo de propiedades. Las combinaciones de productos diseñados se muestran a los clientes en la encuesta de DS para validar la relación entre Kanseis y propiedades del producto.

E.Identificación de la relación entre las palabras Kansei y la síntesis de productos.

Se aplican métodos estadísticos a partir de los resultados de las encuestas de DS para verificar la validez del test y encontrar la relación entre las palabras Kansei y las variables del producto.

\section{Test de fiabilidad alfa de Cronbach}

Se utiliza para medir la fiabilidad de una escala de medida o test, es decir, para medir la ausencia de errores en una prueba, específicamente en la IK se usa para medir la fiabilidad de las pruebas para evaluar la relación entre las palabras Kansei y las propiedades de los productos. El coeficiente alfa de Cronbach generalmente varía de 0 a 1, mientras que un límite inferior generalmente acordado para el coeficiente es 0.70. Sin embargo, en los estudios exploratorios, un valor de 0,60 es aceptable, mientras que los valores de 0,80 o más se consideran satisfactorios en las etapas más avanzadas de la investigación [22]

\section{Síntesis QT1}

La técnica QT1 se usa para encontrar directamente las relaciones existentes entre cada una de las propiedades y niveles que poseen los productos analizados y las palabras Kanseis. [23] recomienda el uso de esta metodología en la IK. En esta metodología se usa un modelo de regresión lineal múltiple, con la particularidad que se usan variables categóricas o dummy, lo cual predice una relación entre las variables categóricas y la respuesta cuantitativa [24].

Para aplicar la síntesis QT1 se codifican las propiedades del producto y sus niveles en variables dummy. Las variables dummy son variables de carácter cualitativo, indican la presencia o ausencia de alguna propiedad o característica del producto. Las variables dummy tomarán el valor 1, si la propiedad está presente en el producto, o 0 en el caso contrario.

Para la codificación del espacio de propiedades se define como “j” a las propiedades y "k" a las sub-propiedades 
de la propiedad “j”, denominándose d_i (j,k) como la codificación del espacio de propiedades, en el cual se tiene (1), en la Fig. 4 se presenta un ejemplo de la codificación definida para un audífono.

$$
d_{i}(j, k)=\left\{\begin{array}{l}
1, \text { si la } s u b-\text { propiedad esta presente en el producto } \\
0, \text { si la } s u b-\text { propiedad no esta presente en el producto }
\end{array}\right.
$$

\begin{tabular}{|c|c|c|c|c|}
\hline \multirow{2}{*}{ Categorias (c) } & \multicolumn{4}{|c|}{ 1. Cuerpo } \\
\hline & Redondo & Rectangular & Paralelog ramo & Ovalado \\
\hline \multirow[b]{2}{*}{ Audífonos } & C11 & C12 & $\mathrm{C} 13$ & C14 \\
\hline & & & & \\
\hline P1 & 1 & 0 & 0 & 0 \\
\hline
\end{tabular}

Fig. 4. Ejemplo de la codificación del espacio de propiedades para la propiedad cuerpo y sus 4 subpropiedades, 1 indica la presencia en el producto de la propiedad, 0 la ausencia de esta propiedad.

\section{F.Identificación de la importancia a través de las palabras Kansei}

Se definen la ecuación de regresión lineal múltiple donde Y i es valoración promedio que obtiene cada Kansei, si existen 7 Kansei, entonces i=7, con esto se obtiene la ecuación (2).

$$
Y_{i}=\beta_{0}+\beta_{1} X_{1 i}+\beta_{2} X_{2 i}+\cdots+\beta_{p} X_{p i}+\epsilon_{i} ; i=1,2, \ldots, n
$$

El modelo de predicción de regresión lineal múltiple usando variable "dummy" del método QT1 se expresa como (3):

$$
Y_{i}=\sum_{j=1}^{r} \sum_{k-1}^{c_{j}} \beta_{j k} \delta_{i}(j, k)
$$

donde $\mathrm{j}=1,2, \ldots, \mathrm{r}$ (siendo $\mathrm{r}$ el número total de propiedades) y $\mathrm{k}=1,2, \ldots, \mathrm{c} \mathrm{j}$ ( es el número total de sub-propiedades para cada propiedad $\mathrm{j}$ )

Para la conexión entre el espacio de propiedades y las palabras Kansei evaluadas a través del DS, se define como $\mathrm{Y}_{\mathrm{i}}$ a el valor obtenido de valoración media de cada Kansei para el clúster definido en el estudio. $\mathrm{B}_{\mathrm{jk}}$ es el valor del coeficiente de la variable "dummy" en la ecuación de regresión lineal múltiple, expresado en (2), este coeficiente muestra la relación de la variable "dummy" con la valoración del Kansei Yi. El objetivo es predecir con exactitud la influencia de las propiedades y sus niveles en los sentimientos de los clientes expresados en los términos Kansei.

\section{G.Determinación de las puntuaciones de contribución}

En esta etapa se analiza la contribución de cada propiedad del diseño a la evaluación total del cada Kansei $Y_{1}$. Las puntuaciones de contribución corresponden a los coeficientes $\mathrm{B}_{\mathrm{jk}}$ de la ecuación de regresión lineal múltiple.

\section{H.Determinación de la estructura del producto}

Luego realizar el proceso de IK, se obtienen las propiedades especificas que debe tener el producto a diseñar. Con la inclusión de estas características se debe desarrollar un producto que cumpla con las especificaciones de 
marca y estilo de la empresa o institución, con todo esto se diseñara un producto que proyecte las emociones y sentimientos que se desean crear en el usuario final. Para esto se debe crear un producto que contenga las sub-propiedades con los valores más altos y produzcan la valoración más alta del Kansei.

\section{I.Evaluación final del producto.}

Finalmente, se presenta un boceto, o diseño 3D del modelo constructivo del producto seleccionado al cliente o usuario final para que vuelva a evaluar este producto según la misma escala de DS usado inicialmente, para confirmar si el producto produce las emociones y sentimientos que se quiere impartir al producto. Se introducen los valores promedio de las evaluaciones de los Kansei en la ecuación de regresión lineal múltiple obtenida a partir de la ecuación (1)

\section{III.METODOLOGÍA.}

\section{A.Selección del dominio}

Para determinar el tipo de producto, mercado objetivo, usuarios y consumidores del audífono de conducción ósea se realizó una encuesta demográfica en la cual se realizaron preguntas sobre la edad del niño, tipo de hipoacusia, género del usuario, lateralidad de la hipoacusia. Se aplicó la encuesta al grupo de padres y usuarios de Microtia Ecuador.

Una vez que se recopilaron estos datos, se efectuó un análisis de Clúster para separar grupos homogéneos de menores de 18 años y mayores de 18 años. Todo el estudio posterior se realizó en base al primer grupo de menores de 18 años. El método de agrupación clúster elegido para el estudio fue el K-means, el cual parte de unos datos iniciales supuestamente homogéneos para encontrar grupos heterogéneos entre ellos, mediante el uso el programa de análisis estadístico Minitab®18. Mediante el análisis clúster y un dendograma se separaron los grupos homogéneos de respuestas.

\section{B.Definición del diferencial semántico.}

En esta etapa se recolectaron las palabras Kansei para describir el dominio que se estableció en el paso anterior con el objeto de calificar el tipo de producto potencial más amplio y confiable caracterizado en el dominio. Para la recolección inicial de palabras se revisaron catálogos, publicidad e información disponibles de audífonos, tanto conducción ósea, como de audífonos normales. También se entrevistó a profesionales del área de terapia de lenguaje para que den sus criterios sobre el diseño que debería tener un audífono. Como el número de palabras iniciales fue amplio, se utilizó un diagrama de afinidad para agrupar palabras que cubran el rango de palabras Kansei definidas. A estas palabras se las definió como las palabras Kansei.

\section{C.Definición del campo de propiedades.}

En esta fase, se identificaron y recogieron las características del producto que describen el dispositivo de conducción ósea caracterizado en el dominio homogéneo luego del análisis clúster. La selección de las características del audífono dio lugar a la construcción de una base de datos con estilos de audífonos, estas se obtuvieron a partir de entrevistas con expertos en audiología, el equipo del proyecto, revisión de bibliografía de audífonos y catálogos de producto. Para cada propiedad del producto se definió una serie de sub-propiedades. Finalmente, el grupo de trabajo definió técnicamente las características más relevantes para el desarrollo del producto. Para esto se generaron 16 bocetos o renders de productos que incluyen una combinación de las sub-propiedades definidas. Estas variaciones de audífonos se obtuvieron considerando las recomendaciones de [8] quienes han establecido lo siguiente: (a) un producto en la selección no puede tener más de una categoría para el mismo artículo; (b) no puede haber productos con categorías iguales para todos los artículos simultáneamente; y (c) debe haber al menos dos unidades de producto seleccionadas en la misma categoría para un artículo dado.

D.Identificación de relaciones entre las palabras Kansei y la síntesis de productos.

Se vinculó las palabras Kansei y las características de los productos a través de una encuesta realizada en línea al grupo Microtia Ecuador, utilizando una escala numérica del 1 al 9, tal como se indica en la Fig. 3, para evaluar la medida en que los productos que representan el dominio están correlacionados con impresiones, sentimientos y emociones. Se les presentaron a los usuarios los 16 diseños generados para que cada uno sea evaluado usando la 
escala de DS. Se aplicó el test de fiabilidad alfa de Cronbach para verificar la fiabilidad de la encuesta, esperando un valor mayor que 0.8

\section{E.Identificación de importancia entre palabras Kansei}

Se aplicó la teoría de cuantificación QT1 para encontrar la relación entre las palabras QT1 y las variantes de producto obtenidas, para esto se codifico el campo de propiedades como variables "dummy". Finalmente se obtuvieron los coeficientes de la ecuación de regresión lineal múltiple de acuerdo con la ecuación (2) usando la herramienta de generación de regresiones lineales del programa Excel.

\section{F.Determinación de las puntuaciones de contribución.}

Al final de aplicar el QTI, se obtuvo el grado de asociación entre cada característica del producto y la palabra Kansei, denominado Category Score (CS), que ayudó para la construcción de un modelo de predicción, para esto se evaluó con la ecuación de regresión lineal múltiple, ver la ecuación (3), la contribución de cada sub-propiedad del producto, usando la codificación de variables "dummy".

\section{G.Selección de la estructura del producto.}

Se seleccionó la estructura del producto que tenga el valor más alto del CS, a partir de esto se seleccionaron las sub-propiedades y se realizó un modelado del producto en el programa Solidworks.

\section{H.Evaluación del producto final}

Se presentó el modelado del producto a un grupo de personas del grupo Microtia Ecuador y se le hizo que lo evalúen con la misma escala de DS que se usó inicialmente empleando las 7 palabras Kansei seleccionadas.

\section{IV.RESULTADOS}

\section{A.Selección del dominio:}

Se obtuvo un total de 16 respuestas a la encuesta demográfica, un resumen de las características demográficas se muestra en la tabla 1.

Tabla 1. Clasificación de los usuarios que respondieron a la encuesta demográfica.

\begin{tabular}{|l|l|c|l|l|}
\hline No & $\begin{array}{l}\text { Lugar de } \\
\text { residencia }\end{array}$ & $\begin{array}{l}\text { Edad del } \\
\text { usuario }\end{array}$ & $\begin{array}{l}\text { Sexo del } \\
\text { usuario }\end{array}$ & $\begin{array}{l}\text { Tipo de } \\
\text { hipoacusia }\end{array}$ \\
\hline 1 & Pelileo & 8 & Masculino & Moderado \\
\hline 2 & Quito & $29^{* *}$ & Masculino & Profunda \\
\hline 3 & Ambato & 13 & Femenino & Severa \\
\hline 4 & El Carmen & $27^{* *}$ & Femenino & Severa \\
\hline 5 & Quito & 2 & Masculino & Severa \\
\hline 6 & Quito & 8 & Masculino & Severa \\
\hline 7 & Cuenca & $45^{* *}$ & Masculino & Severa \\
\hline 8 & Manabí & $39^{* *}$ & Femenino & Moderado \\
\hline 9 & Quito & $42^{* *}$ & Masculino & Severa \\
\hline 10 & Loja & 12 & Masculino & Profunda \\
\hline 11 & Quito & 9 & Femenino & Severa \\
\hline 12 & Quito & 7 & Masculino & Severa \\
\hline 13 & Quito & 12 & Masculino & Moderado \\
\hline 14 & Quito & $26^{* *}$ & Masculino & Moderado \\
\hline 15 & Ambato & 5 & Masculino & Moderado \\
\hline 16 & Quito & 9 & Masculino & Moderado \\
\hline
\end{tabular}

** Grupo que no se tomó en cuenta en el estudio debido a que pertenecen a otro grupo homogéneo. 
A partir de la tabla 1, se obtuvo el dendograma de la Fig. 5, en el cual se observan claramente el grupo de mayores de 18 años y el grupo de menores de 13 años con todos los niveles de hipoacusia y con ambos sexos.

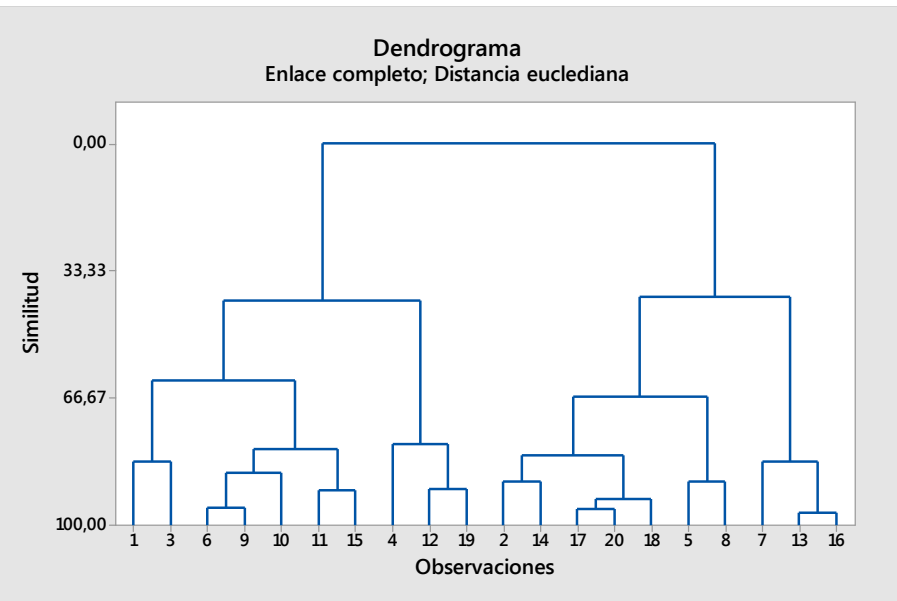

Fig. 5. Dendograma a partir del análisis clúster, se muestran en el segundo nivel 2 grupos definidos.

\section{B.Definición del diferencial semántico.}

A partir del análisis de información sobre productos similares, se obtuvieron 100 palabras Kansei iniciales, las cuales, con el método del diagrama de afinidad, de acuerdo a la Fig. 6a, fueron resumidas a 7 pares de palabras Kansei finales para el estudio, como se indica en la Fig. 6b.

\begin{tabular}{|c|c|c|c|}
\hline \multirow{2}{*}{\multicolumn{3}{|c|}{ Concepto 1: Dinámico, servible, funcional, utilitario, práctico, eficiente. }} & Palabras Kansei \\
\hline & & & Funcional \\
\hline \multicolumn{3}{|c|}{ Concepto 2: Sencillo, fácil, elemental, simple. } & Simple \\
\hline \multicolumn{3}{|c|}{ Concepto 3: Duradero, fuerte, resistente, persistente, seguro. } & Resistente \\
\hline \multicolumn{3}{|c|}{ Concepto 4: Accesible, liviano, ligero. } & Ligero \\
\hline \multicolumn{4}{|c|}{ Concepto 5 : Bello, bonito, atractivo, infantil, cálido, emocionante, original, elegante. } \\
\hline \multicolumn{4}{|c|}{ Concepto 6: Óptimo, moderno, superficial, pequeño. } \\
\hline \multicolumn{3}{|c|}{ Concepto 7: relajante, cómodo, ergonómico, seguro. } & Cómodo \\
\hline $\begin{array}{l}\text { ) Disfuncional - } \\
\text { Funcional }\end{array}$ & Complejo-Simple & No Resistente-Resistente & Pesado - Ligero \\
\hline icómodo-Cómodo & Adulto - infantil & Grande-Pequeño & \\
\hline
\end{tabular}

Fig. 6. a) Diagrama de afinidad b) Definición de las palabras Kansei, las palabras a la izquierda definen el requisito que no es funcional para el cliente y la que está a la derecha define la palabra Kansei funcional..

\section{C.Definición del campo de propiedades.}

Se definieron 4 propiedades principales que debería tener un audífono de conducción ósea: 1) cuerpo del dispositivo de vibración: 2) tipo de ajuste al cráneo; 3) forma del dispositivo de vibración; y 4) color. Dentro de cada una de las propiedades se definieron las sub-propiedades, lo cual se muestra la figura 7. 

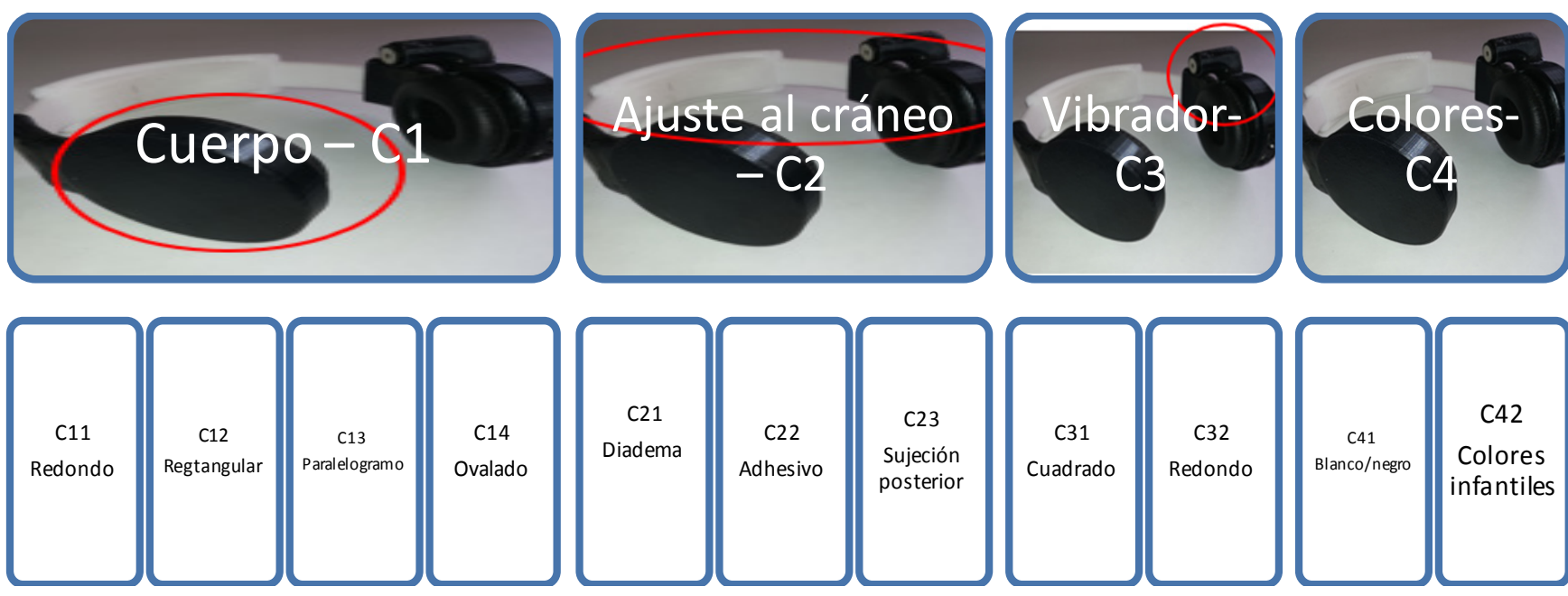

C42

Colores infantiles

Fig. 7. Ejemplo de campo de propiedades y subpropiedades

A partir de la combinación de las sub-propiedades se definieron 16 variantes de productos obtenidas a partir de imágenes renderizadas y modeladas en el programa Solidworks. Las 16 variantes del producto se muestran en la figura 8.

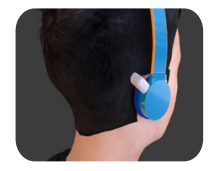

Producto 1 - P1

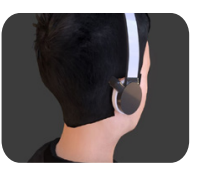

Producto 9- P9

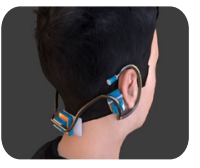

Producto $2-\mathrm{P} 2$

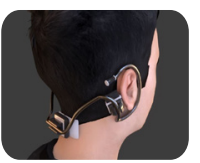

Producto $10-$ P10

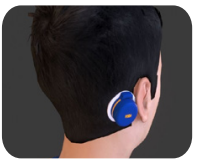

Producto $3-\mathrm{P} 3$

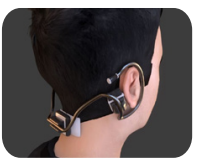

Producto 11 . P11

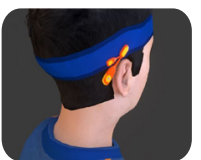

Producto 4 - P4

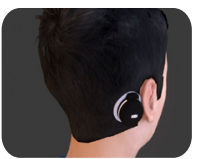

Producto 12 P12

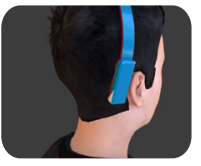

Producto 5 - P5

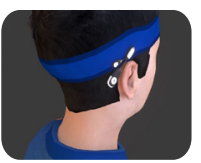

Producto 13 P13

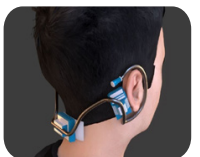

Producto 6-P6

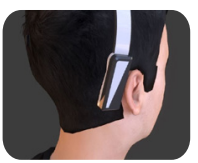

Producto 14 P14

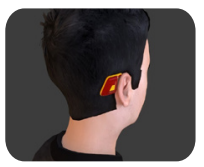

Producto 7-P7

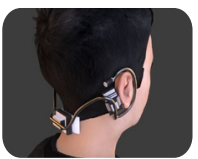

Producto 15 P15

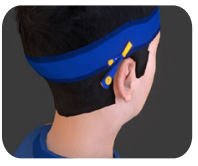

Producto 8-P8

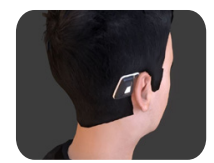

Producto 16 P16

Fig 8. Variantes del producto a partir de la combinación de las sub-propiedades.

\section{D.Identificación de relaciones entre las palabras Kansei y la síntesis de productos.}

Los 16 diseños fueron mostrados a los participantes a través de un cuestionario de Google forms. Se procuró que todas las imágenes presentadas tengan el mismo ángulo de perspectiva. Se pidió a los participantes que evaluaran estos diseños y dieran la puntuación para cada par de palabras. Los promedios de las puntuaciones de pares de palabras de cada muestra se calcularon y se usaron para en el análisis de cuantificación. En la Fig. 9 se muestra un ejemplo de las imágenes presentadas a los encuestados. Las 7 palabras Kansei definidas se les presentaron a los encuestados para que les evalúen en una escala del 1 al 9, similar a la mostrada en figura 3, para evaluar las 16 variantes del producto. 


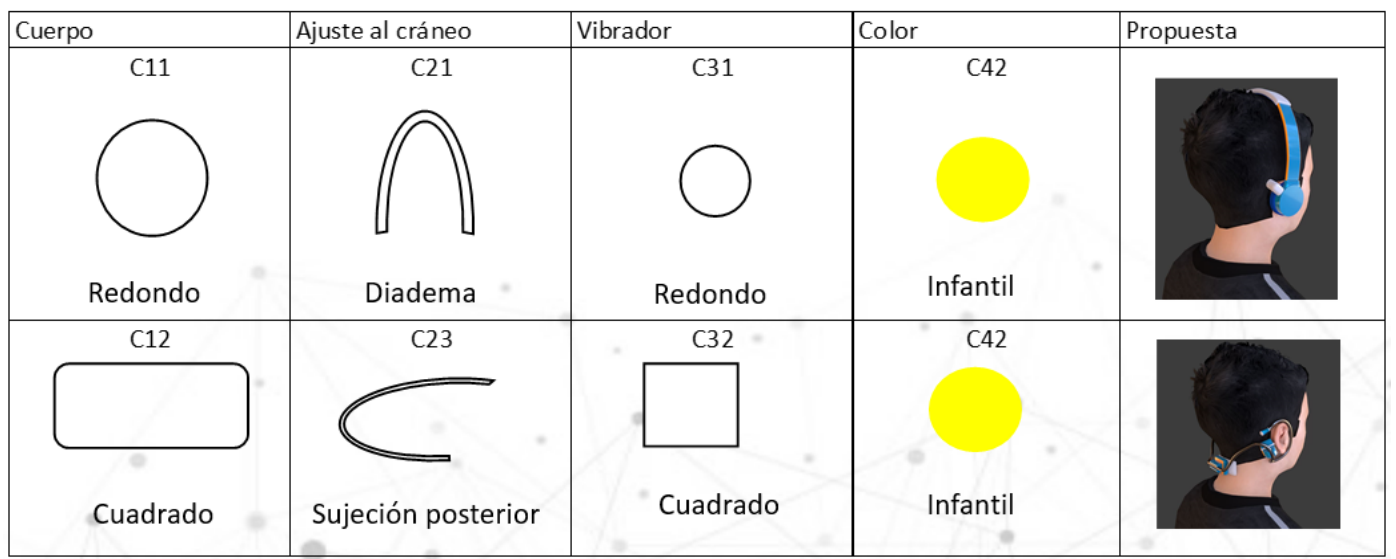

Fig. 9. Muestra de dos de los diseños usados en las encuestas mostradas a los usuarios.

Cada uno de los 16 participantes en la encuesta, evaluaron cada Kansei, con la escala de DS, los resultados la evaluación promedio de los 16 participantes en la encuesta se muestra en la tabla 2. Se usaron también los resultados de la encuesta de DS para aplicar la prueba de fiabilidad alfa de Cronbach, el cual dio un valor de 0.87.

Tabla 2. Resultado de las evaluaciones promedio mediante el DS de 16 variantes de productos para las 7 palabras Kansei.

\begin{tabular}{|c|c|c|c|c|c|c|c|}
\hline & Y1 & Y2 & Y3 & Y4 & Y5 & Y6 & Y7 \\
\hline $\begin{array}{c}\text { Product } \\
\text { o }\end{array}$ & $\begin{array}{c}\text { K1- } \\
\text { Funciona } \\
\text { I }\end{array}$ & $\begin{array}{c}\text { K2- } \\
\text { Simpl } \\
\text { e }\end{array}$ & $\begin{array}{c}\text { K3- } \\
\text { Resistent } \\
\text { e }\end{array}$ & $\begin{array}{c}\text { K4- } \\
\text { Liger } \\
\text { o }\end{array}$ & $\begin{array}{c}\text { K5 - } \\
\text { Infanti } \\
\text { I }\end{array}$ & $\begin{array}{c}\text { K6- } \\
\text { Pequeñ } \\
\text { ○ }\end{array}$ & $\begin{array}{c}\text { K7 - } \\
\text { Cómod } \\
\text { o }\end{array}$ \\
\hline P1 & 4,1 & 2,5 & 3,9 & 2,9 & 2,6 & 2,9 & 3 \\
\hline P2 & 3,4 & 2,6 & 2,8 & 2,6 & 2,4 & 2,4 & 2,6 \\
\hline P3 & 6,1 & 5,5 & 5,4 & 6,1 & 5,6 & 6 & 6 \\
\hline P4 & 5,3 & 4,2 & 4,2 & 4,2 & 3,7 & 3,6 & 3,6 \\
\hline P5 & 4,8 & 4 & 4 & 3,8 & 3,3 & 2,9 & 3,2 \\
\hline P6 & 3,9 & 2,7 & 2,9 & 2,7 & 2,7 & 2,7 & 2,5 \\
\hline P7 & 5,5 & 5,3 & 5 & 5,3 & 4,4 & 4,9 & 4,8 \\
\hline P8 & 4,8 & 4,2 & 4,7 & 4,1 & 4 & 4 & 4 \\
\hline P9 & 4,6 & 3,2 & 3,2 & 3,2 & 3,2 & 3,2 & 3,2 \\
\hline P10 & 3,7 & 2,9 & 2,9 & 2,9 & 2,9 & 2,9 & 2,9 \\
\hline P11 & 5,9 & 5,7 & 5,7 & 5,1 & 5,8 & 5,8 & 5,8 \\
\hline P12 & 3,8 & 3 & 3 & 3 & 3 & 3 & 3 \\
\hline P13 & 4,7 & 3,5 & 3,5 & 3,5 & 3,5 & 3,5 & 3,5 \\
\hline P14 & 4 & 2,8 & 2,8 & 2,8 & 2,8 & 2,8 & 2,8 \\
\hline P15 & 5,4 & 5,3 & 5,3 & 5,1 & 4,7 & 5,4 & 5,4 \\
\hline P16 & 5,4 & 4,2 & 4,2 & 4,2 & 4,2 & 4,2 & 4,2 \\
\hline
\end{tabular}

Análisis de Cuantificación - Síntesis QT1

La codificación de las variables dummy a partir de la presencia o no de las sub-propiedades se muestra en la tabla 3. 
Tabla 3 . Codificación del campo de propiedades con variables dummy.

\begin{tabular}{|c|c|c|c|c|c|c|c|c|c|c|c|}
\hline \multirow{3}{*}{ Categorias (c) } & \multicolumn{11}{|c|}{ Propiedades/Categorias } \\
\hline & \multicolumn{4}{|c|}{ 1. Cuerpo } & \multicolumn{3}{|c|}{ 2. Ajuste al cráneo } & \multicolumn{2}{|c|}{ 3. Vibrador } & \multicolumn{2}{|c|}{ 4. Color } \\
\hline & Redondo & Rectangular & Paralelogramo & Ovalado & Diadema & Adhesivo & Posterior & Redondo & Cuadrado & Negro & Infantil \\
\hline \multirow{2}{*}{\multicolumn{12}{|c|}{ Audífonos }} \\
\hline & & & & & & & & & & & \\
\hline P1 & 1 & 0 & 0 & 0 & 1 & 0 & 0 & 1 & 0 & 0 & 1 \\
\hline P2 & 0 & 1 & 0 & 0 & 0 & 0 & 1 & 0 & 1 & 0 & 1 \\
\hline P3 & 1 & 0 & 0 & 0 & 0 & 1 & 0 & 1 & 0 & 0 & 1 \\
\hline P4 & 0 & 0 & 0 & 1 & 0 & 0 & 1 & 1 & 0 & 0 & 1 \\
\hline P5 & 0 & 1 & 0 & 0 & 1 & 0 & 0 & 0 & 1 & 0 & 1 \\
\hline P6 & 0 & 1 & 0 & 0 & 0 & 0 & 1 & 0 & 1 & 0 & 1 \\
\hline P7 & 0 & 0 & 1 & 0 & 0 & 1 & 0 & 0 & 1 & 0 & 1 \\
\hline P8 & 0 & 1 & 0 & 0 & 0 & 0 & 1 & 0 & 1 & 0 & 1 \\
\hline P9 & 1 & 0 & 0 & 0 & 1 & 0 & 0 & 1 & 0 & 1 & 0 \\
\hline P10 & 0 & 1 & 0 & 0 & 0 & 0 & 1 & 0 & 1 & 1 & 0 \\
\hline P11 & 1 & 0 & 0 & 0 & 0 & 1 & 0 & 1 & 0 & 1 & 0 \\
\hline P12 & 0 & 0 & 0 & 1 & 0 & 0 & 1 & 1 & 0 & 1 & 0 \\
\hline P13 & 0 & 1 & 0 & 0 & 1 & 0 & 0 & 0 & 1 & 1 & 0 \\
\hline P14 & 0 & 1 & 0 & 0 & 0 & 0 & 1 & 0 & 1 & 1 & 0 \\
\hline P15 & 0 & 0 & 1 & 0 & 0 & 1 & 0 & 0 & 1 & 1 & 0 \\
\hline P16 & 0 & 1 & 0 & 0 & 0 & 0 & 1 & 0 & 1 & 1 & 0 \\
\hline
\end{tabular}

Nota: "1" representa la presencia de la propiedad; "2" representa la usencia de la propiedad.

Se usó el programa Excel para sacar la relación en los 7 Kansei (Y_i) usando una regresión lineal múltiple con variables dummy de acuerdo con la ecuación (2). En todos los casos se obtuvo un coeficiente de correlación (CMM) mayor a 0.8, el CMM es un parámetro importante para mostrar la fiabilidad de los resultados estadísticos. Generalmente, el resultado estadístico es fiable y puede ser aceptado si CMM es mayor de 0.7. En este estudio todos los valores de CMM son mayores a 0.77 , lo que indica que todos los resultados son fiables, los CMM se muestras en la tabla 4.

Tabla 4. Coeficientes y rangos obtenidos a través del análisis estadístico de cuantificación QT1 y regresión lineal múltiple con variables "dummy".

\begin{tabular}{|c|c|c|c|c|c|c|c|c|c|c|c|c|c|c|c|c|}
\hline \multirow[b]{3}{*}{ Propiedad } & \multirow[b]{3}{*}{ Subpropiedad } & \multirow[b]{3}{*}{ Cod } & \multirow{2}{*}{\multicolumn{2}{|c|}{$\begin{array}{c}\text { Funcional } \\
\text { K1 }\end{array}$}} & \multicolumn{2}{|c|}{ Simple } & \multicolumn{2}{|c|}{ Resistente } & \multicolumn{2}{|c|}{ Ligero } & \multicolumn{2}{|c|}{ Infantil } & \multicolumn{2}{|c|}{ Pequeño } & \multicolumn{2}{|c|}{ Cómodo } \\
\hline & & & & & K & 2 & K & 3 & & 4 & & 5 & K & 6 & & 7 \\
\hline & & & Score & Range & Score & Range & Score & Range & Score & Range & Score & Range & Score & Range & Score & Range \\
\hline \multirow{4}{*}{ 1. Cuerpo } & Redondo & C11 & -0.75 & \multirow{4}{*}{1.70} & -1.27 & \multirow{4}{*}{2.47} & -0.42 & \multirow{4}{*}{1.02} & -0.98 & \multirow{4}{*}{1.98} & -0.68 & \multirow{4}{*}{2.33} & -0.28 & \multirow{4}{*}{1.18} & -0.38 & \multirow{4}{*}{1.43} \\
\hline & Rectangular & $\mathrm{C} 12$ & 0.95 & & 1.20 & & 0.60 & & 1.00 & & 1.65 & & 0.90 & & 1.05 & \\
\hline & Paralelogramo & $\mathrm{C} 13$ & 0.00 & & 0.00 & & 0.00 & & 0.00 & & 0.00 & & 0.00 & & 0.00 & \\
\hline & Ovalado & C14 & 0.00 & & 0.00 & & 0.00 & & 0.00 & & 0.00 & & 0.00 & & 0.00 & \\
\hline \multirow{3}{*}{ 2. Ajuste al cráneo } & Diadema & $\mathrm{C} 21$ & -1.65 & \multirow{3}{*}{2.20} & -2.75 & \multirow{3}{*}{3.27} & -2.00 & \multirow{3}{*}{2.37} & -2.55 & \multirow{3}{*}{2.98} & -2.80 & \multirow{3}{*}{3.03} & $\mid-2.85$ & \multirow{3}{*}{2.88} & -2.80 & \multirow{3}{*}{2.98} \\
\hline & Adhesivo & $\mathrm{C} 22$ & 0.00 & & 0.00 & & 0.00 & & 0.00 & & 0.00 & & 0.00 & & 0.00 & \\
\hline & Posterior & $\mathrm{C} 23$ & -2.20 & & -3.27 & & -2.37 & & -2.98 & & -3.03 & & -2.88 & & -2.98 & \\
\hline \multirow{2}{*}{ 3. Vibrador } & Redondo & C31 & 1.30 & \multirow{2}{*}{1.30} & 1.57 & \multirow{2}{*}{1.57} & 0.82 & \multirow{2}{*}{0.82} & 1.38 & \multirow{2}{*}{1.38} & 1.83 & \multirow{2}{*}{1.83} & 1.03 & \multirow{2}{*}{1.03} & 1.18 & 1.18 \\
\hline & Cuadrado & $\mathrm{C} 31$ & 0.00 & & 0.00 & & 0.00 & & 0.00 & & 0.00 & & 0.00 & & 0.00 & 1.10 \\
\hline 4. Color & $\begin{array}{l}\text { Negro } \\
\end{array}$ & C41 & -0.05 & 0.05 & -0.05 & 0.05 & -0.29 & 0.29 & -0.24 & 0.24 & 0.17 & 0.17 & 0.18 & 0.18 & 0.14 & 0.14 \\
\hline 4. Color & Infantil & $\mathrm{C} 42$ & 0.00 & & 0.00 & & 0.00 & & 0.00 & & 0.00 & & 0.00 & & 0.00 & \\
\hline & & Constante & & 48 & 5. & 33 & 5. & 29 & & 32 & & 46 & 5. & 06 & & 03 \\
\hline & & CMM & & 77 & 0. & 88 & 0. & 84 & & 88 & & 89 & 0. & 92 & & 92 \\
\hline
\end{tabular}

\section{E.Determinación de las puntuaciones de contribución.}

La columna score de la tabla 4 explica el grado de correlación entre los elementos de diseño y el par de palabras Kansei. Por ejemplo, tomar el par de palabras funcional-disfuncional a través de las 4 subpropiedades de la propiedad cuerpo, el valor redondo obtiene el valor más bajo, -0.75 , lo que índica que se acerca más a la palabra Kansei 
disfuncional. Contrariamente, la forma de cuerpo rectangular, obtiene el valor más alto, 0.95 , lo que muestra que este diseño es más funcional. La ecuación de la regresión líneal múltipe para el Kansei 1 (funcional) de da según la ecuación (4). Las otras categorías de propiedades y valores de los Kansei pueden analizarse con el mismo método.

$$
K_{1}=0,95 C_{12}+0 C_{22}+1,30 C_{31}+0 C_{41}
$$

La columna "range" de la tabla 4 para cada Kansei puede explicar la influencia de cada propiedad sobre el juicio de percepción de la emoción del usuario, este valor "range" se calcula restando el valor mayor de la columna "score" menos el valor menor. Tomemos como ejemplo las palabras "funcional-disfuncional"; el orden en descenso del valor "range" es ajuste al cráneo (2.2), cuerpo (1.70), vibrador (1.30) y color(0.05). Se puede inferir que el ajuste al cráneo tiene la mayor influencia en el juicio de percepción, el segundo es el cuerpo, y el color tiene menos influencia.

\section{F.Selección de la estructura del producto.}

La estructura seleccionada a partir de seleccionar los valores más altos de los coefiencientes de la ecuación de regresión lineal múltiple (score) se indican en la Fig. 10a, en el cual el diseño incorpora un diseño rectangular, con adhesivo, con vibrador redondo y de color negro Fig 10b. En la Fig. 10c. se muestra el diseño del audífono puesto en la cabeza del usuario.

a)

\begin{tabular}{|c|c|c|}
\hline Kansei & Propiedad & Subpropiedad \\
\hline & 1. Cuerpo & Rectangular \\
\cline { 2 - 3 } & 2. Ajuste al cráneo & Adhesivo \\
\cline { 2 - 3 } $\begin{array}{c}\text { Funcional, } \\
\text { Simple, } \\
\text { Resistente, } \\
\text { Ligero, Infantil, } \\
\text { Pequeño, } \\
\text { Cómodo }\end{array}$ & 3. Vibrador & Redondo \\
\cline { 2 - 3 } & 4. Color & Negro \\
\hline
\end{tabular}

b)

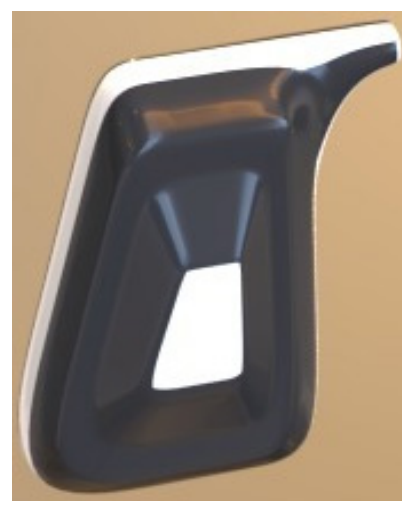

c)

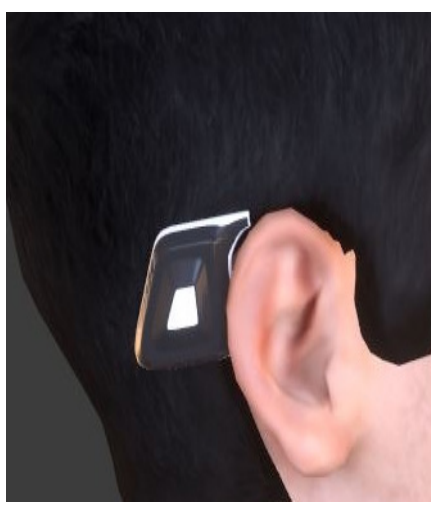

Fig. 10. a) Combinación de sub-propiedades que obtuvieron el mayor puntaje b) Modelado del producto final c) Modelado del producto final sobre el hueso mastoides del usuario.

\section{G.Evaluación del producto final}

La Fig. 11a muestra la evaluación que obtuvo el diseño final presentado (Fig.11) a un grupo de personas. En esta evaluación se obtuvo respuesta de 37 personas del grupo Microtia Ecuador, se obtienen una evaluación promedio de 6.5 sobre 9 puntos de la escala de DS, mientras que la predicción mediante cada una de las ecuaciones de regresión lineal múltiple se obtiene un valor promedio de 5.15 sobre 9 , lo que indica que el diseño produce las emociones indicadas por el conjunto de palabras Kansei, ver Fig. $11 \mathrm{~b}$. 


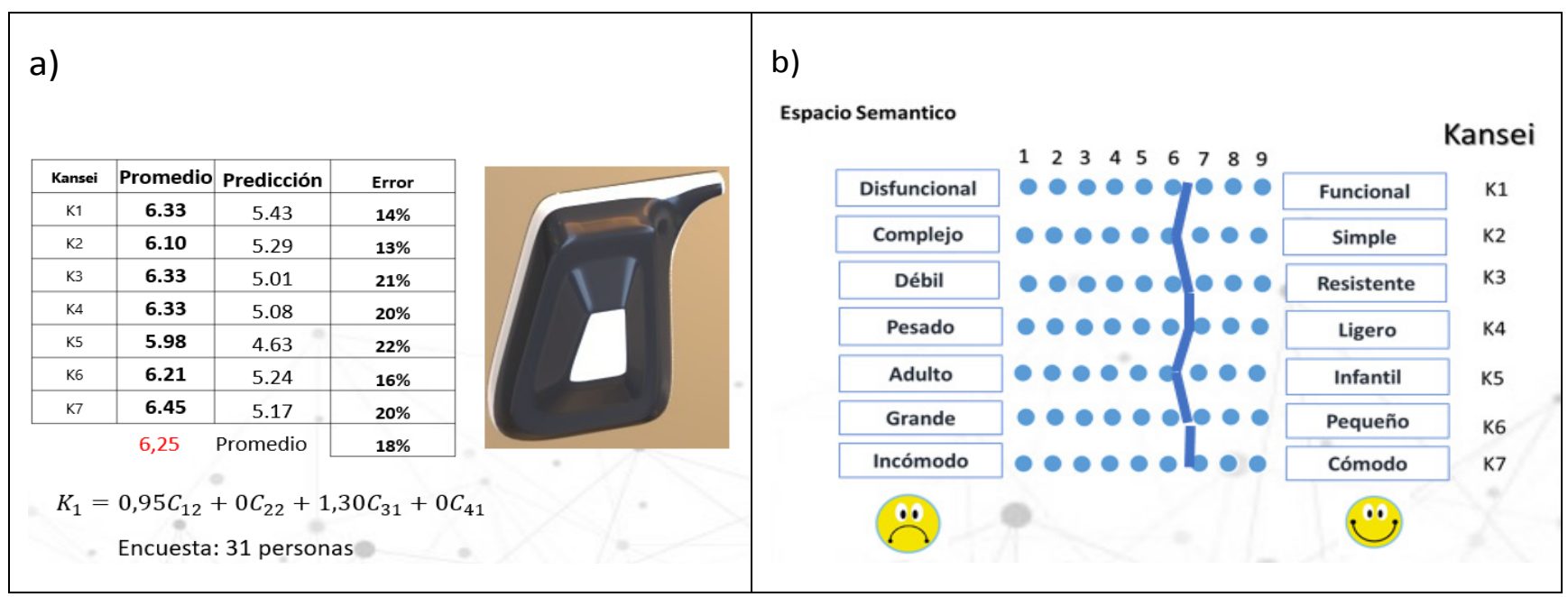

Fig. 11. a) Resultados de la evaluación del diseño seleccionado por usuarios usando los Kansei. b) Escala de diferencial semántico para el diseño final.

\section{V.CONCLUSIONES}

1)Se ha logrado crear un concepto de un dispositivo para audición por conducción ósea y accionamiento cutáneo que permite causar emociones positivas en el usuario final, aplicando la ingeniería Kansei, superior a la predicción realizada por la regresión lineal múltiple, este tipo de evaluación final evaluando el diseño con otra muestra de posibles usuarios, no se ha encontrado en otros estudios de IK.

2)Todas las emociones medidas mediante la encuesta del producto final seleccionado muestran reacciones positivas sobre el diseño, en un valor sobre sobre la escala de 9.

3)Esta metodología permite el desarrollo de un producto que será bien aceptado por el mercado objetivo, solo realizando modelos conceptuales, sin el costo que implica la construcción de un modelo físico y el posible rechazo futuro de los usuarios.

\section{RECONOCIMIENTO}

Se agradece a la Comisión de Investigación Formativa y a los Proyectos Semilla de la Universidad Central del Ecuador, por su apoyo con la dirección y financiamiento para el desarrollo de esta investigación.

\section{REFERENCIAS}

[1]El Telégrafo, «La discapacidad auditiva afecta a 360 millones de personas en el mundo,» 28 septiembre 2017. [2]J. Nazer, G. Lay-Son y L. Cifuentes, «Prevalencia al nacimiento demicrotia-anotia. Maternidad delHospital Clínico de la Universidadde Chile, período 1983-2005,» Revista médica de Chile, 2006.

[3]M. E. Arévalo Molina y D. E. Sánchez Navarrete, «Identificación de factores de riesgo de microtía en niños atendidos en el servicio de otorrinolaringología del Hospital Pediátrico Baca Ortiz, en el período 2010 a 2014,» Universidad Católica del Ecuador, Quito, 2016.

[4]Organización Mundial de la Salud, «Organización Mundial de la Salud,» 15 marzo 2019. [En línea]. Available: https://www.who.int/es/news-room/fact-sheets/detail/deafness-and-hearing-loss.

[5]F. Coello, H. Cuevas y E. Andrade, «El tamizaje auditivo neonatal en Ecuador, un compromiso ineludible,» pp. 177-184, 2016.

[6]M. F. Torres Saravia, «Estudio comparativo del nivel de desarrollo neuropsicológico en niños y niñas con deficiencia,» Quito, 2013.

[7]R. Dauman, B. Carbonnièr, V. Soriano, S. Berger-Lautissier, J. Bouyé, E. Debruge, G. Coriat y J.-P. Bébéar, «Implantes cocleares en el adulto y en el niño,» Elsevier, pp. 1-13, 2000.

[8]M. J. Lavilla Martín de Valmaseda, L. Cavalle Garrido, A. Huarte Irujo, F. Núnez Batalla, M. Manrique Rodriguez, Á. Ramos Macías, C. d. P. Vernetta, E. Gil-Carcedo Sañudo, L. Lassaleta, I. Sánchez-Cuadrado, J. M. Espinosa Sánchez, Á. Batuecas Caletrio y C. Cenjor Español, «Guía clínica sobre implantes de conducción de vía 
ósea,» Elsevier, pp. 761-766, 2017.

[9]M. Nagamachi y A. M. Lokman, Innovations of Kansei Engineering, Tokyo: CRC Taylor \& Francis Group, 2003.

[10]I. Griha y T. Isa, «Kansei Engineering Approach in Software Interface Design Indra,» JOURNAL OF SCIENCE INNOVARE, vol. 01, nº 01, pp. 22-26, 2018.

[11]I. Mañero y A. García, «El diseño Kansei y su aplicacion en la invención de una muleta de ayuda a la movilidad,» Revista Anales ICAI, vol. 1, nº 1, pp. 1-17, 2010.

[12]Y. Wang y Y. Chen, «The Kansei Research on the Style of Women's Overcoats,» Multiconference on "Computational Engineering in Systems Applications"(CESA),, pp. 431-436, 2006.

[13]L. S. Martinez, La percepción del confort. Análisis de los parámetros de diseño y ambientales mediante Ingeniería Kansei: Aplicación a la biblioteca de Ingeniería del Diseño (UPV), Valencia: UPV, 2011.

[14]P. Rodríguez de Andrés, Ingeniería Kansei y su aplicación en el diseño emocional de bibliotecas, Valencia: UPV - Universidad Politécnica de Valencia, 2013.

[15]Fundación Prodintec, Diseño Afectivo e Ingeniería Kansei - Guía Metodológica, Gijón, Asturias, España: Centro Tecnológico para el Diseño y la Producción Industrial en Asturias Parque, 2011.

[16]S. Schütte, Engineering Emotional Values in Product Design, Linköping, Sweden: Linköpings Universitet Department of Mechanical Engineering, 2005.

[17]A. M. Lokman, «Design and Emotion: The Kansei Engineering,» Faculty of Computer and Mathematical Sciences, Universiti Teknologi MARA (UiTM) Malaysia, vol. 1, nº 1, pp. 1-14, 2010.

[18]S. Khalid, Biometrics and Kansei Engineering, New York: Springer Science+Business Media, 2012.

[19]L. D. Ferreira y D. C. Amaral, «A full example of technical procedure to application of the Kansei Engineering,» Product: Management \& Development, vol. 13 , no 2, pp. 103-118, 2015.

[20]Y. Matsubara y M. Nagamachi, «An application og image procesing technology in Kansei Engineering,» Ergonomie et design, vol. 4, pp. 123-126, 1994.

[21]J. E. Bauer, . G. L. Duffy y R. T. Westcott, The Quality Improvement Handbook, Milwaukee: ASQ - Quality Press, 2006.

[22]M. Nagamachi, Kansei/Affective Engineering and History of Kansei/Affective Engineering in the World, Boca Raton: Taylor and Francis Group, LLC, 2011.

[23]E. Mooi, M. Sarstedt y I. Mooi-Reci, Springer Texts in Business and Economics Market Research The Process, Data, and Methods Using Stata, Singapore: Springer Nature, 2018.

[24]S. T. W. Schütte, J. Eklund, J. R. Axelsson y M. Nagamachi, «Concepts, Methods and Tools in Kansei Engineering,» Theoretical Issues in Ergonomics Science, $n^{\circ}$ 1, 2004.

[25]G. Smith, Essential Statistics, Regression, and Econometrics., Elsevier Inc. All, 2015.

\section{RESUMEN CURRICULAR}

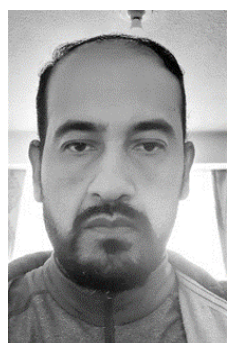

Ing. Mauricio Fuentes MSc, Ingeniero Mecánico, Máster en Gestión de la Calidad y Productividad, Docente en de la Carrera de Ingeniería en Diseño Industrial de la Universidad de la Central del Ecuador.

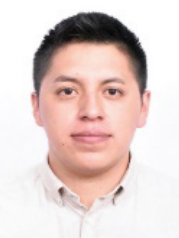

Tnlogo. Henry Yánez, Tecnólogo en Emergencias Médicas, Egresado de la Carrera de Ingeniería en Diseño Industrial de la Universidad de la Central del Ecuador. 


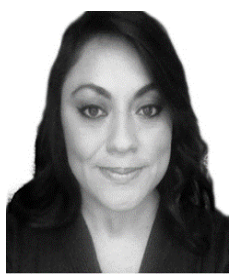

Mgt. Andrea Villalobos, Ingeniera en Diseño Gráfico Publicitario. Maestría en Arquitectura de la Información en la Pontificia Universidad Católica del Ecuador, Sede Ambato. Profesora investigadora en la Universidad Central del Ecuador.

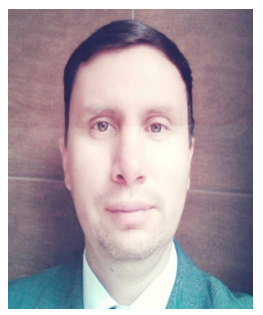

Ing. Diego Veintimilla, MBA mención Gestión de Proyectos (EPN), Diplomado en Calidad y Productividad, Ing. de Sistemas Informáticos y de Computación (EPN). Asesor en Emprendimiento, Innovación y Gestión Empresarial. Coordinador de Proyectos-Solidarity Tech. 